\title{
El Jardín Hidrobotánico Jorge Hernández Camacho de Caucasia: Un recurso didáctico para la enseñanza y el aprendizaje del concepto Ecosistema
}

\author{
The Garden of Hidrobotánico Jorge Hernandez Camacho Caucasia: A didactic resource \\ for teaching and learning of the ecosystem concept \\ Yuri Yadith Benítez Díaz', Tatiana Lorena Ortiz Álvarez ${ }^{1}$, Ledis Mańa Sampayo Londoño ${ }^{1}$, Juan \\ Diego Cardona Restrepo ${ }^{2}$
}

\begin{abstract}
${ }^{1}$ Universidad de Antioquia. ${ }^{2}$ Universidad de Antioquia - Universidad Internacional de Andalucía Grupo de Investigación Innovaciencia yubedi26@yahoo.com , torenaalvarez@gmail.com , sampylo@gmail.com, jdcardona@ayura.udea.edu.co
\end{abstract}

\section{Resumen}

En este trabajo de investigación monográfica, se indaga por las concepciones alternativas de un grupo de estudiantes referentes al concepto de ecosistema y sobre cómo estas concepciones evolucionan luego de que, los estudiantes participan en el desarrollo de una Unidad Didáctica ejecutada en la reserva natural Jardín Hidrobotánico Jorge Hemández Camacho de Caucasia. La propuesta de enseñanza se ha estructurado alrededor del Cido Didáctico, siguiendo los fundamentos de la Metacognición y Autorregulación del Aprendizaje. Es de anotar que al momento de presentar este artículo para evaluación de pares, el trabajo se encuentra en la segunda fase correspondiente a la aplicación y recolección de la información, por lo que no es posible dar cuenta de los resultados obtenidos, más si para la fecha de celebración del evento.

Palabras clave

Educación Ambiental, Concepciones Altemativas, Ciclo de Aprendizaje, Metacognición y Autorregulación del Aprendizaje, Ecosistema, Jardín Botánico y Reserva Natural.

\section{Abstract}

In this research monograph, it explores the alternative conceptions of a group of students regarding the concept of ecosystem and how these concepts evolve after that, students participate in the development of a teaching unit implemented in the natural reserve Hidrobotánico Garden Jorge Hernandez Camacho of Caucasia. The proposed school is structured around the Teaching Cyde, following the basics of Metacognition and Self- Learning. It should be noted that at the time of submitting this article for peer evaluation, the work is in the second phase for the implementation and collection of information, which is not possible to account for the results, if for timing of the event.

\section{Keywords}

Environmental education, altemative conceptions, learning cyde, metacognition and selflearning ecosystems, botanical gardens and nature reserve. 


\section{Introducción}

La educación ambiental es un espacio que le posibilita al ser humano la interrelación de otros saberes a través de la comprensión y análisis de factores y situaciones ambientales; que buscan la preservación, conservación y aprovechamiento del medio. La educación ambiental tiene sus raíces desde tiempos remotos en los que se enseñaba al hombre a vivir en armonía con la naturaleza. Sin embargo a finales de los años 1960 y principio de 1970 esta comienza a ser difundida con tal designación: Educación Ambiental (de ahora en adelante EA). Con el afloramiento de esta disciplina se buscaba hacer frente a los problemas ambientales.

La EA le facilita al ser humano relacionarse con su entomo, reconociendo valores, adarando conceptos que le permiten fomentar actitudes y aptitudes criticas y reflexivas, con el fin de comprender y apreciar la realidad concreta de las interrelaciones entre el hombre, su cultura y su medio biofísico. Esta relación y compresión de la naturaleza es necesaria encaminarla desde una metodología que favorezca el aprendizaje de los estudiante como es el ciclo de aprendizaje; cido que parte de las concepciones alternativas, que muchas veces están presentes en ellos, cuando al incorporar una nueva información, activan en su memoria los conocimientos relacionados con fenómenos para los cuales no tienen explicaciones cientúficas construidas, estableciendo conexiones e interpretaciones de la nueva información en función del conocimiento previo existente. En relación con esto Bermúdez y De Longhi (2008) tras una reflexión teórica sostienen que la ecología debe actuar como vigilante epistemológico de la mayoría de los contenidos que se trabajan bajo el enfoque de Educación Ambiental para no caer en un reduccionismo conceptual y curricular basado en la mera discusión que entablan docente y alumnos sólo desde sus referentes cotidianos y además se debe considerar las concepciones alternativas de los estudiantes que conlleve en el curríaulo el tratamiento de de los fundamentos científicos de temáticas ecológicas.

Es así, como con este trabajo que tiene como titulo El Jardín Hidrobotánico Jorge Hernández Camacho de Caucasia: un recurso didáctico para la enseñanza y el aprendizaje del concepto de ecosistema se busca enseñar desde la EA el concepto ecosistema, teniendo en cuenta los cidos de aprendizaje y las concepciones altemativas de los estudiantes, integrando espacios vivos o reservas naturales como el Jardín Hidrobotánico del Bajo Cauca, a las actividades cotidianas del aula en el marco del desarrollo de una unidad didáctica, con el fin de conocer la evolución de las concepciones acerca del concepto Ecosistema en los estudiantes del grado $5^{\circ}$ A de la Institución Educativa Escuela Normal Superior del Bajo Cauca. En donde los actores (estudiantes) puedan obtener fundamentos ecológicos, conciencia conceptual, deseo de investigación, evaluación de problemas, la capacidad de acción y el reconocimiento de sus conocimientos y sus avances (parte fundamental de la metacognición y autorregulación del aprendizaje).

Respondiendo así a la invitación de cambiar la rutina y los cánones ortodoxos del trabajo tradicional en el aula; para hacer de las dases unas prácticas pedagógicas amenas en el que la motivación del estudiante se mantenga y se refleje en la evolución de las concepciones altemativas por unas nuevas concepciones. Por ello se considera pertinente la interacción de los estudiantes con el entomo natural. De acuerdo con esto Tirado y López (1994) luego de una investigación sobre la enseñanza de la biología en México conduyen que el rendimiento del sistema educativo nacional es muy pobre, debido a la manera en que se presenta el conocimiento al estudiante. Se enseña información de manera encidopédica, aislada, desarticulada de un contexto general de integración que le de congruencia que permita desarrollar un significado (y significación global) holístico.

Con el fin de no continuar con este problema, proponen la presentación de contenidos a partir del modelo curricular ofreciendo varias ventajas, la más importante quizá sea que el alumno no vea la necesidad de memorizar simples conocimientos, sino que le permita encontrar una explicación de sus propios orígenes, de su naturaleza, del entormo que lo rodea; con lo que se transforma la 
significación del conocimiento escolar y es más probable que pueda estimular su interés por el conocimiento. Análogo a esto Fuentes et al (2006) en una investigación sobre la transversalidad aurricular en la enseñanza de la educación ambiental, sugieren que sólo con un proceso de construcción de conocimientos a partir de las experiencias previas de los alumnos y en relación con el entomo, se podrá alcanzar un proceso de aprendizaje de calidad, que sea deseado, sentido, comprendido y esté abierto a las transformaciones por parte de los alumnos, pero en armonía con docentes, directivos, actores políticos y económicos, así como con la comunidad.

La propuesta de incorporar desde la EA las reservas naturales al sistema educativo contribuye a que "se oriente modificar actitudes, proporcionar nuevos conocimientos y criterios, promover la participación directa y la practica comunitaria. Es decir a la búsqueda de una solución a los problemas existentes en el mundo" (UNESCO, 2005).

Convirtiéndose así la EA en la herramienta social para afrontar las problemáticas presentes ocasionadas por el deterioro del ambiente; asegurando de tal forma, para las generaciones futuras un ambiente sano y propicio para el desarrollo sostenible de los reaursos. Además la EA es importante en la formación del individuo puesto que abre una perspectiva vital, a través del manejo de las diversas variables de la dinámica de la vida y logra ubicar al individuo como ser natural y a la vez como un ser social (Maldonado, 2005). Pues a tavés del crecimiento y el desarrollo, el hombre va captando conocimiento y transformándolo en beneficio para él, ello es precisamente lo que ocurre con la EA ya que por medio de ella el ser humano crea pautas de comportamiento y moldea su conducta como ser social.

Así mismo, como propone Navarro (1997) el interés de la EA no es solamente propiciar algunas modificaciones en las enseñanzas escolares, sino suscitar nuevos conocimientos fundamentales [...] la creación de nuevas relaciones entre todos los miembros de la sociedad y su repercusión sobre el medio ambiente [...] por ello incumbe a la educación y formación ambiental -con instrumentos y estrategias de acción fundamentales de la integración y del cambio social y cultural- definir objetivos y recurrir a medios nuevos que permitan a los individuos ser más consientes, más responsables y estar mejor preparados, con un cambio de actitud para hacer frente a los retos de la preservación de la calidad del ambiente y de la vida. Un ejemplo de los instrumentos mencionados es el aprovechamiento del Jardín Hidrobotánico en el marco del desarrollo de una unidad didáctica, enfocada desde el cido de aprendizaje. Develándose la interrelación de dos aspectos, un campo abierto o natural que posibilita enseñar y aprender de una manera práctica y directa; y por otro lado, una unidad didáctica que hace las veces de derrotero de la teoría y la experiencia.

La idea de esta investigación, no surge de un problema como tal; sino de un interés de conocimiento que yace en el hecho de que, en la actualidad el medio ambiente afronta un sin número de problemáticas, como: la deforestación, la contaminación de las aguas, el desequilibrio en los ecosistemas y muchos más que si seguińamos mencionando pasańamos años luz sin acabar. Esto ha sido debido a la presión que la actividad humana ejerce sobre el medio, lo cual ha llevado a debilitar la interacción entre los componentes del sistema ambiental, entendiéndose este como un tejido de relaciones en el que la cultura, en su proceso de construcción permanente, presenta elementos que actúan como mecanismos de adaptación, para la interacción sociedad-naturaleza, de igual forma es posible percibir un medio cada vez mas vulnerado, un mayor agotamiento de los recursos y otras problemáticas ambientales que se sitúan no en los síntomas, sino en el centro mismo de la actividad humana contextualizada por una forma cultural; ya que cada cultura modifica su ambiente natural y en consecuencia en determinado tipo de problemas ambientales (Mendoza 2001). Por tanto, cada componente cultural y social es influenciado por los avances de la ciencia y la tecnología sumados a toda acción del hombre que reperaute indudablemente en la desarticulación del sistema ambiental. De igual forma Galhano - Alves (2004) en un estudio sobre las representaciones culturales que tiene una comunidad que habita en el Parque Natural 
Montesinho (Portugal) sobre el jabalí encuentra que las personas tienen poco conocimiento de la ecología y la utilidad social de esta especie, solo lo perciben como una presa para ser cazada induso muchas personas piensan que estos deben ser exterminados. Para hacer frente a esta situación sugiere que la EA y la participación en la conservación podría aportar a un mejor equilibrio de los factores, ecológicos, culturales y económicos entre los seres humanos y el Jabalí. Si desde la EA y el trabajo monográfico se ayuda a los estudiantes a que tomen conciencia sobre la importancia de conservar el Jardín Hidrobotánico y las relaciones de sus diferentes componentes, podríamos contribuir a su recuperación, conservación y sostenibilidad.

Es importante el estudio de los ecosistemas como concepto estructurante de las dinámicas naturales entre diversos factores o componentes del ambiente, teniendo en cuenta de que el hombre, en su afán por la civilización y desarrollo, agota los recursos naturales al extremo de extinguirlos. Pero no se puede perder de vista que el hombre hace parte de él, necesita de él, y está en la obligación de cuidarlo y preservarlo para garantizar su propia supervivencia y de las generaciones futuras. Por ello se hace necesario que la educación se pronuncie ante la comprensión de los ecosistemas; permitiendo al estudiante reconocer cada uno de sus componentes, las relaciones que se pueden establecer entre el hombre y la naturaleza y, las dinámicas propias del entomo natural, las alteraciones del ecosistema por el modo de vida, la cultura, la sociedad y el desarrollo; objetivo que se puede lograr integrando en las propuestas educativas el trabajo de campo en espacios o entomos naturales. Tal cual como lo propone Canton (1991) en una experiencia exitosa,

Granja - Escuela La Campaza, en la que se puso de relieve la estrecha interrelación entre el hombre (estudiantes) y el medio ambiente configurado en una estancia rural, propiciando el dinamismo de objetivos de contenidos de enseñanza que pretenden ofrecer a los alumnos generalmente proveniente de medios urbanos un tipo de enseñanza donde el medio rural 0 agrario se convierte en cercano, acogedor, entretenido como fuentes de saberes y disfrutes, que posibilita el contacto con la naturaleza, con los animales, las gentes etc. Aunque nuestro interés no se desarrolla dentro de un área rural son importantes estos aportes desde el punto de vista de una enseñanza en el que el sujeto adquiere o modela su conocimiento en la relación con la naturaleza.

Paralelamente a esto Garcáa (2003) en un estudio afimma que para la construcción en el aula de la noción de ecosistemas se propone la integración didáctica de las aportaciones de la ecología entendida como disciplina cientúfica y como cosmovisión- con las concepciones del alumnado. Dicha integración lleva a una hipótesis de progresión que articula y orienta el correspondiente proceso de investigación. Los diversos modelos didácticos para la enseñanza de las ciencias naturales y la Educación Ambiental, entre ellos el modelo de investigación en la escuela permiten conectar un entramado de saberes que le da la posibilidad al estudiante permear sus conocimientos de diversas formas, ya sea teórica o experimentalmente. Como es el caso de estudiar en reservas naturales, entendidas como espacios naturales para la conservación y sostenibilidad de los ecosistemas, comunidades y elementos biológicos. Donde el maestro de ciencias naturales en su planeación le dé este sentido y propicie actividades en la que se halle la relación de lo natural, con lo cultural y lo social. Como en el caso de, García (2005) que en un estudio plantea concretamente que en el área de Ciencias Naturales son numerosas las actividades de distinta índole que se pueden llevar a cabo para lograr los objetivos propuestos en las programaciones. Independientemente de los propios recursos de los departamentos o del entomo más o menos cercano a los centros. Pues la enseñanza de las ciencias naturales no puede estar limitada a espacio cerrados y orientada bajo elementos artificiales y abstractos; sino más bien tangibles, observables y manipulables. 


\section{Desarrollo}

Dos preguntas guían este trabajo de investigación. La primera de ellas, ¿Cuáles son las concepciones (Antes, durante, después) del desarrollo de una unidad didáctica sobre el concepto de ecosistema de un grupo de estudiantes del grado $5^{\circ}$ de la Institución Educativa Escuela Normal Superior del Bajo Cauca? y una segunda, ¿Cómo evolucionan las concepciones sobre el concepto de ecosistema en un grupo de estudiantes del grado $5^{\circ}$ de la Institución Educativa Escuela Normal Superior del Bajo Cauca, aprovechando el Jardín Hidrobotánico en el marco del desarrollo de una unidad didáctica?

Estas concepciones se evaluaran con base a las siguientes categorías a priori (reconociendo que se tendrán en cuenta también las posibles categorías emergentes).

Estas serán denotadas desde la C1 a la C8.

$>\quad$ C1 Definición de ecosistema

$>\quad$ C2 Actitudes de los estudiantes cuando desarrollan actividades en una reserva

$>$ natural

$>\quad$ C3 Esquemas que realizan los estudiantes antes de visitar un ecosistema natural y

$>$ luego de visitarlo

$>\quad$ C4 Elementos que el estudiante cree que hacen parte de un ecosistema

$>$ C5 Interacciones de los humanos y los ecosistemas

> C6 Aprovechamiento de los ecosistemas

C C7 Estrategias para la reauperación y conservación de los ecosistemas

$>$ C8 Evolución conceptual

Con el fin de alcanzar los objetivos propuestos, éste trabajo de investigación se sustenta bajo la perspectiva Metodológica Cualitativa (Estudio de caso descriptivo), enfocado desde el paradigma socio-critico debido a que este permite identificar las concepciones de los estudiantes antes del proceso de intervención y luego conocer la evolución de éstas en relación con el concepto de ecosistema; emancipando al sujeto para que analice la realidad critica del entomo; reconozca los conocimientos intuitivos que su cultura le ha legado sobre la temática, las acciones que tiene con el medio natural y los valores que sustentan las relaciones con el ambiente; para que luego se pueda hacer consciente de los conocimientos que la escuela le ofrece y pueda compararlos con su cultura de base.

La población que se escogió para el desarrollo de este trabajo es un grupo de $\mathbf{4 0}$ estudiantes de $5^{0}$ grado de la educación Básica Primaria de la Institución Educativa Escuela Normal Superior del Bajo Cauca del municipio de Caucasia. La muestra está conformada por un grupo de 9 estudiantes, que se han seleccionado con la previa aceptación de las directivas, padre de familia de la institución y la disposición de los estudiantes para participar como sujetos de la investigación.

\section{Sobre la Unidad Didáctica diseñada}

La Unidad Didáctica denominada "Aprendiendo con la Naturaleza" se ha diseñado para aplicarse entre los meses de mayo y julio del año en curso. Se proyecta para un total de diez sesiones de dase. Se propones como objetivo central: Al finalizar la unidad didáctica el estudiante del grado $5^{0}$ de la Institución Educativa Escuela Normal Superior del Bajo Cauca deberá reconocer la evolución de sus concepciones sobre el concepto ecosistema partiendo de sus ideas altemativas hasta la construcción de un nuevo conocimiento que acerca del ambiente desde una perspectiva sistémica.

Se establecen algunos objetivos espećficos delimitados como: objetivos conceptuales, procedimentales y actitudinales: 
Tabla No. 1. Objetivos espećficos de la Unidad Didáctica

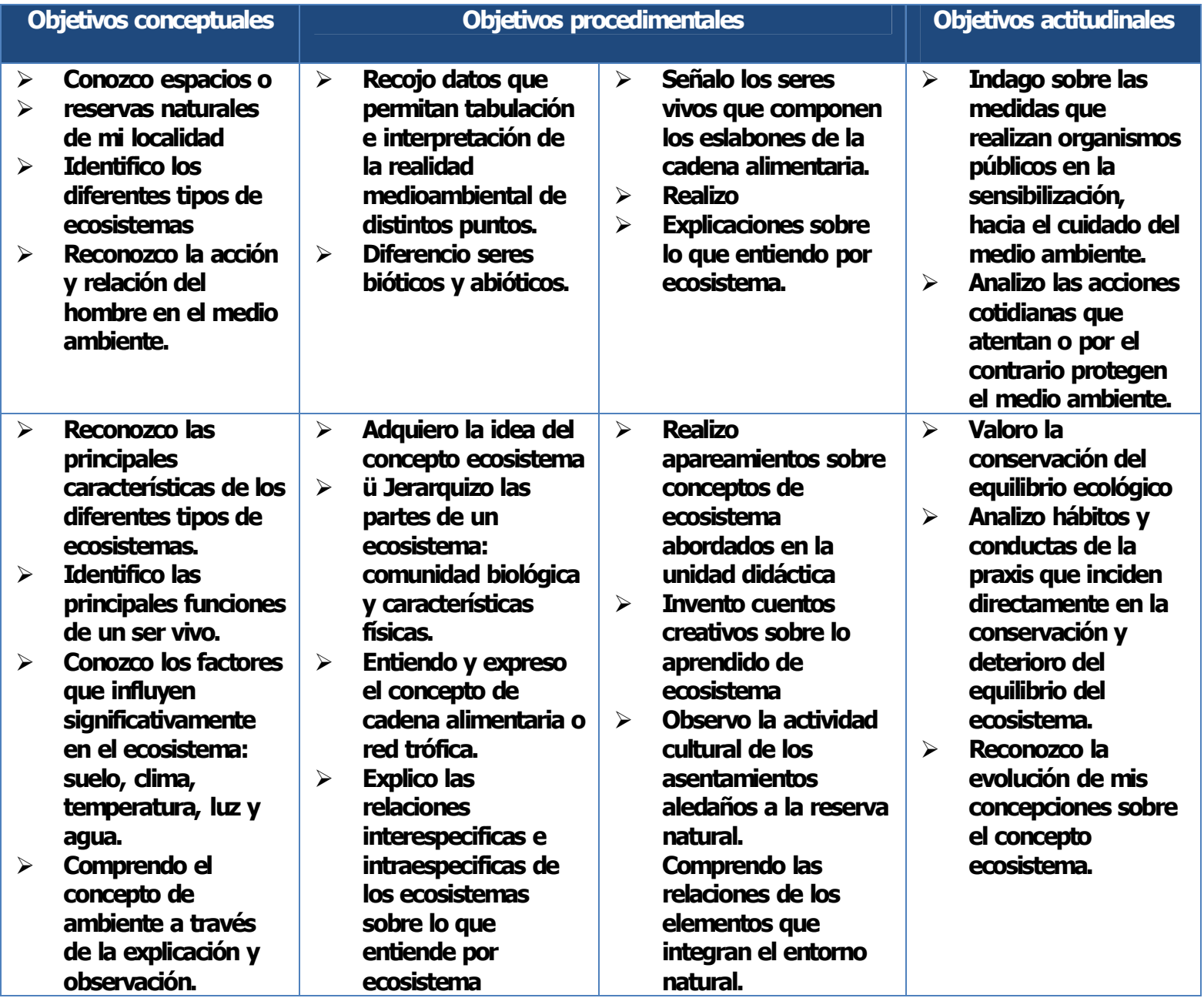

Las actividades de la unidad Didáctica se han organizado en auatro tipologías o fases:

I. Ideas Previas: consta de tres actividades. Me autoregulo y debato sobre el concepto ecosistema, la elaboración de una maqueta para develar la representación de un ecosistema por parte de los alumnos participantes y una visita al Jardín Hidrobotánico mediante una guía de observación que contiene un cuestionario con preguntas abiertas.

II. Introducción de Nuevos Conocimientos: tres visitas guiadas al Jardín Hidrobotánico donde el profesor explicará y presentará algunos temas relacionados con el ecosistema en un escenario natural. Igualmente se han propuesto algunas actividades y preguntas orientadoras, relacionadas con la actividad inicial.

III. Estructuración y Síntesis: dos actividades permiten la estructuración y síntesis de los nuevos conocimientos. La primera en donde se dibujaran y expresaran artísticamente los componentes del ecosistema. La segunda es un trabajo practico que consiste en una visita guiada al Caño Atascoso (para comparar espacios contaminados por el hombre versus espacios conservados).

IV. Aplicación: la principal actividad de aplicación es la reelaboración de la maqueta inicial, induyendo los nuevos conocimientos, en especial, sobre las relaciones del hombre con la naturaleza. La segunda actividad es la última visita realizada al Jardín Hidrobotánico, está a su vez 
consta de dos partes, una en la que se hace un apareamiento de conceptos y definiciones relacionadas con el ecosistema. Otra en la que los estudiantes inventan un cuento sobre la conservación y aprovechamiento del ecosistema.

Para la recolección de la información además se utilizarán técnicas e instrumentos como las entrevistas abiertas semiestructuradas, la observación, el diario de campo, materiales audiovisuales (grabaciones, videos y fotos) y artefactos grupales (maquetas) ü Entrevista abierta semiestructurada: Durante el desarrollo de la unidad didáctica, estas serán realizadas a los estudiantes, a través de preguntas abiertas. Organizadas así: en la primera y segunda actividad de exploración, en la sesión tres de la actividad de introducción de conocimientos, en la segunda actividad de estructuración y en la tercera actividad de aplicación. Mediante las cuales se busca saber cómo los estudiantes conciben el concepto general de ecosistema, antes, durante y después del desarrollo de la unidad didáctica y de igual forma ver cómo evolucionan sus concepciones alternativas.

> La Observación: Se observará detalladamente a los estudiantes en sus actitudes y valores, asumidos frente a los organismos y recursos hallados en la reserva durante la visita y el recorrido por el Jardín Hidrobótanico. Espećficamente en la tercera actividad de exploración. (Ver anexo 1)

> El diario de campo: Esta herramienta nos servirá para registrar la información más relevante de las actividades realizadas y reflexionar a la vez sobre las mismas.

$>$ Materiales y artefactos: Para este tipo de instrumento se utilizaran video cámaras, mp4, maqueta, dibujos y esquemas.

> Documentos escritos: Se le pedirá a los estudiantes que elaboren un quento sobre la forma como se encuentran organizados los seres vivos en ecosistemas. Sin olvidar induir el auidado, conservación y aprovechamiento de los mismos. Con ello pretendemos ver que concepciones alternativas continúan arraigadas y auales han evolucionado.

El análisis e interpretación de la información se basa en cuatro técnicas:

- Análisis del discurso de los participantes antes, durante y después del desarrollo de la unidad didáctica.

> Las redes sistémicas para categorizar y analizar la información en jerarquías. con la cual se busca realizar una diagnosis o prognosis en los sujetos antes de iniciar un tema o unidad didáctica.

> Cuestionarios KPSI, con el cual se busca que los estudiantes autorregulen su aprendizaje, diseñado por Tamir y Luneta (1978).

$>$ El formato de Tablas propuesta por Martínez (1998) que consta de de dos columnas: la primera donde se efectúa la categorización, la recategorización y las anotaciones especiales y, en la segunda se transcriben literalmente las respuestas de los estudiantes; marcando con códigos los diferentes interlocutores desde el A1 hasta el A6 (Ver tabla No. 2).

\section{Resultados obtenidos}

Hasta la fecha se han realizado las actividades planeadas en la fase de exploración, la cual consta de tres actividades. Un debate sobre el concepto ecosistema, la elaboración de una maqueta para develar la representación de un ecosistema por parte de los alumnos participantes y una visita al Jardín Hidrobotánico mediante una guía de observación que contiene un cuestionario con preguntas abiertas. En esta primera fase se ha recolectado la información de las actividades programadas y en este momento se hallan en proceso de sistematización y análisis, por lo cual, los resultados que se induyen a continuación son parciales. 
Definición de ecosistema y elementos que el estudiante cree que hacen parte de un ecosistema

Para esta categoría se plantean dos instrumentos un cuestionario KPSI y una entrevista con las mismas preguntas del cuestionario anterior, en donde los dos nos permitieron recoger los siguientes resultados. La entrevista demuestra que los estudiantes tienen un poco conocimiento del tema y que confunden muchos conceptos tanto de biología como de ecología. Solo 3 de 9 estudiantes tienen una aproximación al concepto de ecosistema y 5 de 9 estudiantes no son daros en sus argumentos. En cuanto a los resultados del cuestionario KPSI su valoración se resume en el aradro No 1.

Esquemas que realizan los estudiantes antes de visitar un ecosistema natural y luego de visitarlo

Para recoger los datos de esta categoría se utilizo el instrumento materiales y artefactos, espeć́ficamente la elaboración de una maqueta sobre lo que los estudiantes consideran es un ecosistema y para analizar dichos resultados planteamos una red sistémica. Con esta pudimos ver que los estudiantes representan componentes del ecosistema como vegetales (arboles, hierbas y arbustos), animales terrestres y materia no viva como agua, rocas y montañas. Además 4 de 9 estudiantes incorporaron elementos no propios del ecosistema como calles, edificios, carros y aeropuertos. Por último se observa que no representan relaciones entre los seres vivos y los factores abióticos.

Actitudes de los estudiantes cuando desarrollan actividades en una reserva natural

Luego de una visita al jardín Hidrobotánico pudimos observar que los estudiantes son poco respetuosos con los recursos naturales y los seres vivos, teniendo en cuenta que arrancaron plantas indiscriminadamente sin ningún objetivo, lanzaron piedras a árboles frutales, mataron animalitos como hormigas y mariposas. Además presentaron un comportamiento inadecuado según las instrucciones que orientó el guía de la reserva 


\section{Cuestionario tipo KPSI de ideas relacionado con el aprendizaje del concepto ecosistema}

\section{VALORANDO LO QUE SE}

Nombre

Fecha:

Veamos a continuación una lista de unos cuantos aspectos que se pueden estudiar sobre el ecosistema.

Al lado de cada uno marcar con una $(x)$ según la siguiente tabla:

$$
\begin{aligned}
& 1=\text { no lo sé } \\
& 2=10 \text { sé un poco } \\
& 3=\text { lo sé bastante bien } \\
& 4=10 \text { sé bien } \\
& 5=10 \text { puede explicar a un amigo } 0 \text { amiga }
\end{aligned}
$$

\begin{tabular}{|c|c|c|c|c|c|}
\hline \multirow{2}{*}{ PREGUNTAS } & \multicolumn{5}{|c|}{ CODIGOS } \\
\hline & 1 & 2 & 3 & 4 & 5 \\
\hline ¿Qué es un ecosistema? & 1 & 4 & 1 & 2 & 1 \\
\hline $\begin{array}{l}\text { ¿Cuáles son los componentes de un } \\
\text { ecosistema? }\end{array}$ & 3 & 2 & 1 & 1 & 2 \\
\hline $\begin{array}{l}\text { ¿Cómo está estructurado u organizado un } \\
\text { ecosistema? }\end{array}$ & 3 & 4 & 1 & 1 & 0 \\
\hline $\begin{array}{l}\text { ¿Oaáles son las relaciones que se dan } \\
\text { dentro del ecosistema? }\end{array}$ & 4 & 2 & 3 & 0 & 0 \\
\hline $\begin{array}{l}\text { ¿Qué es la cadena alimenticia o red } \\
\text { trófica? }\end{array}$ & 0 & 0 & 5 & 2 & 2 \\
\hline $\begin{array}{l}\text { ¿Quál es la diferencia entre biótico y } \\
\text { abiótico? }\end{array}$ & 8 & 0 & 0 & 1 & 0 \\
\hline ¿Qué es especie? & 1 & 4 & 2 & 2 & 0 \\
\hline ¿Qué es comunidad? & 0 & 2 & 5 & 1 & 1 \\
\hline ¿Qué es población? & 1 & 3 & 2 & 2 & 1 \\
\hline
\end{tabular}

Cuestionario a 9 estudiantes

\section{Conclusiones}

Esta investigación se ha propuesto en tres momentos. Inicialmente con un rastreo bibliográfico y planteamiento del problema. Seguidamente el marco teórico, el diseño metodológico y la elaboración de la propuesta (unidad didáctica). Por último se consolidará la sistematización, análisis de los resultados y la redacción del informe final; por tanto hasta el momento solo podemos hablar de resultados parciales a la investigación, teniendo en cuenta que estamos comenzando el tercer momento. Sin embargo, para la fecha de celebración del evento ya contamos con todos los resultados y sus respectivos análisis. 
No obstante, podemos afirmar, que los diversos modelos didácticos para la enseñanza de las ciencias naturales y la educación ambiental, entre ellos el modelo de investigación en la escuela permiten conectar un entramado de saberes que le da la posibilidad al estudiante permear sus conocimientos de diversas formas, ya sea teórica o experimentalmente como es el caso de un aprendizaje colaborativo en entomos naturales.

Según el cuestionario KPSI, conduimos que, algunos los estudiantes al momento de autorregularse, sobre lo que conocen de ecosistema reflejan poca capacidad y sinceridad para reconocer lo que realmente saben sobre el tema; pues se ubican en una escala muy alta, que se deja ver en contraposición al momentos definir los conceptos, otros son consientes y reconocen no saber nada.

En cuanto al esquema que representan el ecosistema permite considerar que los conceptos que manejan de ecosistema no son muy daros y que les falta mayor profundidad en el tema, pues confunden temas propios de la ecología con otros de biología.

\section{Bibliografía}

BERMUDEZ, G Y DE LONGHI, A. (2008). La Educación Ambiental y la Ecología como ciencia. Una discusión necesaria para la enseñanza. Revista Electrónica de Enseñanza de las Ciencias 7(2), 275 $-297$

CANTON, I (1991). Granja - Escuela La Campaza. Revista interuniversitaria de formación del profesorado. No 11, $113-124$.

CARRASCOSA, J. (2005). El problema de las concepciones alternativas en la actualidad (parte I). Análisis sobre las causas que las originan y/o mantienen. Revista Eureka sobre Enseñanza $y$ Divulgación de las Ciencias, 2(2), 183-208.

CARRASCOSA, J. (2005). El problema de las concepciones alternativas en la actualidad (parte I). Análisis sobre las causas que las originan y/o mantienen. Revista Eureka sobre Enseñanza $y$ Divulgación de las Ciencias, 2(2), pp. 183-208.

CASTILLO, A. (2003). Comunicación para el manejo de Ecosistemas. Tópicos en Educación Ambiental 3 (9), 58-71.

GALHANO-ALVES, J.P. (2004). Man and wild boar: A study in Montesinho Natural Park, Portugal. Galemys: Boletín informativo de la sociedad española para la conservación y estudio de los mamíferos, 16, No extra 1, 223-230.

GARCÍA, A. (2005). El jardín botánico como recurso didáctico. Revista Eureka sobre Enseñanza y Divulgación de las Ciencias, 2, 209-217.

MALDONADO, H. A. (2005) La educación Ambiental como herramienta social. Revista venezolana de geografía y su enserianza, 10 (1). pp. 61 - 67.

SANMARTí, N. (1998). La evaluación de los aprendizajes, (adaptado del capítulo 2 del libro: Gairin, J. \& Sanmartí, N. (1998) La evaluación institucional. Ministerio Educación. Argentina.

UNESCO (1997). Educación para un futuro sostenible: Una visión transdisciplinaria para una acción concertada. Thessalonike: UNESCO-Gobiemo de Grecia 